\title{
Modulation of the faecal microbiome of healthy adult dogs by inclusion of potato fibre in the diet
}

\author{
Matthew R. Panasevich ${ }^{1}$, Katherine R. Kerr ${ }^{1}$, Ryan N. Dilger ${ }^{1}$, George C. Fahey Jr ${ }^{1}$, \\ Laetitia Guérin-Deremaux ${ }^{2}$, Gary L. Lynch ${ }^{3}$, Daniel Wils ${ }^{2}$, Jan S. Suchodolski ${ }^{4}$, Jörg M. Steiner ${ }^{4}$, \\ Scot E. Dowd ${ }^{5}$ and Kelly S. Swanson ${ }^{1 *}$ \\ ${ }^{1}$ Department of Animal Sciences, University of Illinois, 1207 West Gregory Drive, Urbana, IL 61801, USA \\ ${ }^{2}$ Roquette Frères, Biology and Nutrition Department, Rue de la Haute Loge, Lestrem 62080, France \\ ${ }^{3}$ Roquette America, Inc., 2211 Innovation Drive, Geneva, IL 60134, USA \\ ${ }^{4}$ Gastrointestinal Laboratory, Texas AEM University, College Station, TX 77843, USA \\ ${ }^{5}$ MR DNA Molecular Research LP, 503 Clovis Road, Shallowater, TX 79363, USA
}

(Submitted 3 March 2014 - Final revision received 21 August 2014 - Accepted 10 September 2014 - First published online 24 November 2014)

\begin{abstract}
Inclusion of fermentable fibres in the diet can have an impact on the hindgut microbiome and provide numerous health benefits to the host. Potato fibre (PF), a co-product of potato starch isolation, has a favourable chemical composition of pectins, resistant and digestible starch, cellulose, and hemicelluloses. The objective of the present study was to evaluate the effect of increasing dietary PF concentrations on the faecal microbiome of healthy adult dogs. Fresh faecal samples were collected from ten female dogs with hound bloodlines $(6.13$ (SEM 0.17$)$ years; 22.0 (SEM 2.1$) \mathrm{kg}$ ) fed five test diets containing graded concentrations of PF $(0,1.5,3,4.5$ or $6 \%$ as-fed; Roquette Frères) in a replicated $5 \times 5$ Latin square design. Extraction of DNA was followed by amplification of the V4-V6 variable region of the 16S rRNA gene using barcoded primers. Sequences were classified into taxonomic levels using Basic Local Alignment Search Tool (BLASTn) against a curated GreenGenes database. Inclusion of PF increased $(P<0 \cdot 05)$ the faecal proportions of Firmicutes, while those of Fusobacteria decreased $(P<0.05)$. Similar shifts were observed at the genus level and were confirmed by quantitative PCR (qPCR) analysis. With increasing concentrations of PF, faecal proportions of Faecalibacterium increased $(P<0 \cdot 05)$. Post hoc Pearson's correlation analysis showed positive $(P<0.05)$ correlations with Bifidobacterium spp. and butyrate production and Lactobacillus spp. concentrations. Overall, increases in the proportion of Faecalibacterium (not Lactobacillus/ Bifidobacterium, as confirmed by qPCR analysis) and faecal SCFA concentrations with increasing dietary PF concentrations suggest that $\mathrm{PF}$ is a possible prebiotic fibre.
\end{abstract}

Key words: Potato fibre: Faecal microbiome: Dogs

The gastrointestinal tract of humans and animals is occupied with a dense and diverse population of bacteria that are known to influence the health of the host. Changes in the host's microbiome are affected by a number of factors including genetics ${ }^{(1)}$, age ${ }^{(2)}$, geographical location ${ }^{(3)}$ and especially diet $^{(4)}$. In particular, fermentable fibres have been known to modulate favourably the hindgut microbiome of both humans and animals ${ }^{(4,5)}$. However, the effects of the dietary inclusion of graded concentrations of fermentable potato fibre (PF) on the hindgut microbiome have not been investigated.

$\mathrm{PF}$ is a co-product of potato starch manufacture with the potential to beneficially modulate the hindgut microbiome. It is high in fibres such as hemicelluloses, cellulose and pectin, and non-fibre components such as starch, oligopeptides and free amino acids ${ }^{(6)}$. The monomeric composition of destarched PF consists mainly of galactose, galacturonic acid, arabinose, and rhamnose, which indicates that the cell wall material is made up primarily of homogalacturonan and rhamnogalacturonan I with long galactan side chains ${ }^{(7)}$. Limited research has investigated the use of PF as a dietary fibre source in dogs and humans. In vitro data using human faecal inoculum indicate that solubilised PF fractions were fermentable and increased the number of bifidobacteria and lactobacilli more than fructo-oligosaccharides ${ }^{(7-9)}$.

The present study is reported in two parts: (1) to evaluate the effects of $\mathrm{PF}$ on in vitro fermentation, and in vivo effects of $\mathrm{PF}$ on nutrient digestibility, faecal fermentation end

Abbreviations: PF, potato fibre; qPCR, quantitative PCR

*Corresponding author: Dr K. S. Swanson, fax +1 217333 7861, email ksswanso@illinois.edu 
products and consistency, and (2) to assess the modulatory effects of dietary PF on the faecal microbiome. Our laboratory previously published data from part 1 , which concluded that $\mathrm{PF}$ was a moderately fermentable fibre both in vitro and in vivo, and had no detrimental effects on total nutrient digestibility or faecal consistency ${ }^{(10)}$. Part 2 is presented here to investigate the effects of graded concentrations of dietary $\mathrm{PF}$ on the modulation of the dog faecal microbiome using $16 \mathrm{~S}$ rRNA gene pyrosequencing. Based on our previous in vitro results (i.e. increased faecal acetate, propionate and butyrate concentrations, and decreased faecal $\mathrm{pH})^{(10)}$, we predicted the changes in faecal SCFA concentrations that would be associated with consequent shifts in the microbiome. Based on previous studies involving dietary fibre in pets and human subjects ${ }^{(4,5)}$, we predicted that at the phylum level, an increase in the faecal proportion of Firmicutes and a decrease in the faecal proportion of Fusobacteria would occur. More specifically, we also predicted the increases in the proportions of Bifidobacterium and other SCFA-producing genera (e.g. Blautia, Lachnospira and Faecalibacterium) with increasing dietary PF concentrations.

\section{Materials and methods}

\section{Animals and diets}

The animal protocol was approved by the University of Illinois Animal Care and Use Committee, and the study was conducted at the Edward R. Madigan Laboratory at the
University of Illinois. A total of ten female dogs with hound bloodlines (6.13 (SEM 0.17) years; 22.0 (SEM 2.1) kg) were used in a replicated $5 \times 5$ Latin square design to test five diets during five time periods. The dogs were housed individually $(2.4 \times 1.2 \mathrm{~m}$ kennels $)$ in two temperature- and light-controlled rooms. Fresh water was available ad libitum.

Overall, five diets containing graded concentrations of PF $(0,1.5,3,4.5$ or $6 \%$ as-is; Roquette Frères) were formulated to exceed the recommended nutrient allowance values of adult dogs (Table 1), with target concentrations of approximately $26 \%$ crude protein and $15 \%$ crude fat (as-is basis) $^{(11)}$. To achieve similar dietary fibre and protein concentrations among the diets, PF was added at the expense of cellulose (Solka Floc; International Fiber Corporation) and brewer's rice. The remainder of the dry, extruded, kibble diet was composed of the following ingredients: low-ash poultry by-product meal; poultry fat; brewer's rice; ground maize; vitamin and mineral premixes. The diets were mixed and extruded at the Kansas State University's Bioprocessing and Industrial Value-Added Program facility (Manhattan, KS, USA) under the supervision of Pet Food and Ingredient Technology, Inc. (Topeka, KS, USA).

Each of the five, $14 \mathrm{~d}$ periods consisted of a $10 \mathrm{~d}$ dietary adaptation phase followed by $4 \mathrm{~d}$ of faecal collection. The dogs were offered $155 \mathrm{~g}$ diet twice daily (08.00 and 17.00 hours) to meet the recommended energy needs of the dog based on the estimated metabolisable energy content of the diet. Fresh faecal samples were collected at the same time as

Table 1. Ingredients and chemical composition of the experimental diets fed to dogs

\begin{tabular}{|c|c|c|c|c|c|}
\hline \multirow[b]{2}{*}{ Items } & \multicolumn{5}{|c|}{$\% \mathrm{PF}^{*}$} \\
\hline & 0 & 1.5 & 3 & $4 \cdot 5$ & 6 \\
\hline \multicolumn{6}{|l|}{ Ingredients (\% as-fed) } \\
\hline Brewer's rice & 46.55 & $45 \cdot 90$ & $45 \cdot 25$ & 44.60 & 43.95 \\
\hline Low-ash poultry by-product meal & $25 \cdot 50$ & $25 \cdot 50$ & $25 \cdot 50$ & $25 \cdot 50$ & 25.50 \\
\hline Maize, yellow, ground & $12 \cdot 00$ & $12 \cdot 00$ & $12 \cdot 00$ & $12 \cdot 00$ & $12 \cdot 00$ \\
\hline Poultry fat & 8.00 & 8.00 & 8.00 & 8.00 & 8.00 \\
\hline PF† & 0.00 & 1.50 & 3.00 & 4.50 & $6 \cdot 00$ \\
\hline Cellulose & $6 \cdot 00$ & $5 \cdot 15$ & $4 \cdot 30$ & 3.45 & $2 \cdot 60$ \\
\hline Salt & 0.70 & 0.70 & 0.70 & 0.70 & 0.70 \\
\hline Potassium chloride & 0.56 & 0.56 & 0.56 & 0.56 & 0.56 \\
\hline Chromic oxide & 0.20 & 0.20 & 0.20 & 0.20 & 0.20 \\
\hline Mineral mix§ & 0.18 & 0.18 & 0.18 & 0.18 & 0.18 \\
\hline Vitamin mix\| & $0 \cdot 18$ & $0 \cdot 18$ & $0 \cdot 18$ & 0.18 & 0.18 \\
\hline Choline chloride- $50 \%$ & 0.13 & 0.13 & 0.13 & 0.13 & 0.13 \\
\hline \multicolumn{6}{|l|}{ Chemical composition (\% DM) } \\
\hline DM & 95.75 & 95.65 & 95.51 & 95.45 & 95.29 \\
\hline Organic matter & 94.21 & 94.36 & 94.24 & 94.07 & $94 \cdot 20$ \\
\hline Crude protein & $25 \cdot 10$ & 24.78 & 24.91 & $25 \cdot 20$ & $25 \cdot 14$ \\
\hline Acid-hydrolysed fat & 13.45 & 14.51 & 14.48 & $14 \cdot 17$ & 13.05 \\
\hline Total dietary fibre & $10 \cdot 79$ & $11 \cdot 24$ & 11.43 & $11 \cdot 17$ & 11.35 \\
\hline Gross energy $(\mathrm{kJ} / \mathrm{g})$ & 20.79 & 20.96 & 20.92 & $20 \cdot 96$ & 20.67 \\
\hline
\end{tabular}

PF, potato fibre.

* Diet formulations contained raw PF at the specified concentrations; diets were extruded to produce the food provided to the dogs

† Roquette Frères, Lestrem, France.

$\ddagger$ Solka-Floc; International Fiber Corporation, North Tonawanda, NY, USA.

$\S$ Provided per kg diet: $\mathrm{Mn}\left(\right.$ as $\left.\mathrm{MnSO}_{4}\right), 66.00 \mathrm{mg} ; \mathrm{Fe}\left(\right.$ as $\left.\mathrm{FeSO}_{4}\right), 120 \mathrm{mg} ; \mathrm{Cu}\left(\right.$ as $\left.\mathrm{CuSO}_{4}\right), 18.00 \mathrm{mg}$; Co (as $\mathrm{CoSO}_{4}$ ), $1.20 \mathrm{mg} ; \mathrm{Zn}\left(\right.$ as $\left.\mathrm{ZnSO}_{4}\right), 240 \mathrm{mg}$; I (as KI), $1.80 \mathrm{mg}$; Se (as $\mathrm{Na}_{2} \mathrm{SeO}_{3}$ ), $0.24 \mathrm{mg}$.

\|Provided per $\mathrm{kg}$ diet: vitamin A, $5.28 \mathrm{mg}$; vitamin $D_{3}, 0.04 \mathrm{mg}$; vitamin $\mathrm{E}, 120.00 \mathrm{mg}$; vitamin $\mathrm{K}$, $0.88 \mathrm{mg}$; thiamin, $4.40 \mathrm{mg}$; riboflavin, $5.72 \mathrm{mg}$; pantothenic acid, $22.00 \mathrm{mg}$; niacin, $39.60 \mathrm{mg}$; pyridoxine, $3.52 \mathrm{mg}$; biotin, $0.13 \mathrm{mg}$; folic acid, $0.44 \mathrm{mg}$; vitamin $\mathrm{B}_{12}, 0.11 \mathrm{mg}$. 
reported previously ${ }^{(10)}$. On day 11 , a fresh subsample of the faeces was collected for analysis of microbial populations. Subsamples were collected in $2 \mathrm{ml}$ cryovials within $15 \mathrm{~min}$ of defecation, snap-frozen in liquid $\mathrm{N}_{2}$, and stored at $-80^{\circ} \mathrm{C}$ until DNA extraction. Faecal butyrate concentration was determined according to previous methods ${ }^{(10)}$. Briefly, one aliquot of approximately $2 \mathrm{~g}$ was collected and placed in approximately $2 \mathrm{ml}$ of $2 \mathrm{~m}-\mathrm{HCl}$ and analysed using a gas chromatograph (Hewlett-Packard 5890A Series II; Hewlett Packard Company) and a glass column $(180 \mathrm{~cm} \times 4 \mathrm{~mm}$ inner diameter), packed with $10 \% \mathrm{SP}-1200 / 1 \% \mathrm{H}_{3} \mathrm{PO}_{4}$ on $80 / 100+$ mesh Chromosorb WAW (Supelco, Inc.). $\mathrm{N}_{2}$ was the carrier gas with a flow rate of $75 \mathrm{ml} / \mathrm{min}$. Oven, detector and injector temperatures were 125,175 and $180^{\circ} \mathrm{C}$, respectively.

\section{Faecal DNA extraction and 454 pyrosequencing}

Bacterial DNA was extracted ${ }^{(12)}$ using the MO BIO PowerSoil $^{\mathrm{TM}}$ Kit (MO BIO Laboratories). Concentration of the extracted DNA was quantified using a NanoDrop ND-1000 spectrophotometer (NanoDrop Technologies) and diluted to $10 \mathrm{ng} / \mathrm{ml}$.

Quality of DNA was assessed by electrophoresis using precast agarose gels (E-Gel ${ }^{\circledR}$ EX Gel $1 \%$; Invitrogen). Amplification of a $600 \mathrm{bp}$ sequence of the $\mathrm{V} 4-\mathrm{V} 6$ variable region of the $16 \mathrm{~S}$ rRNA gene was done using barcoded primers ${ }^{(13)}$. Amplicons from PCR were then further purified using AMPure XP beads (Beckman Coulter, Inc.). Amplicons were combined in equimolar ratios to create a DNA pool that was used for pyrosequencing. Quality of DNA from amplicon pools was assessed before pyrosequencing using a 2100 Bioanalyzer (Agilent Technologies). Pyrosequencing was performed at the W. M. Keck Center for Biotechnology at the University of Illinois using a 454 Genome Sequencer and FLX titanium reagents (Roche Applied Science).

\section{Bioinformatics}

High-quality (quality value $>25$ ) sequence data derived from the sequencing process were processed using a proprietary analysis pipeline (www.mrdnalab.com) and as described previously ${ }^{(14-20)}$. Briefly, the sequences were depleted of barcodes and primers, short sequences $(<200 \mathrm{bp})$, sequences with ambiguous base calls, and sequences with homopolymer runs exceeding $6 \mathrm{bp}$. The sequences then were denoised and chimeras were removed. Operational taxonomic units (OTU) were defined after removal of singleton sequences and clustering at 3\% divergence ( $97 \%$ similarity). Then, OTU were taxonomically classified using Basic Local Alignment Search Tool (BLASTn) against a curated GreenGenes database $^{(21)}$ and compiled into each taxonomic level into both 'counts' and 'percentage' files.

\section{Quantitative $P C R$}

Quantitative PCR (qPCR) was performed according to previous methods ${ }^{(22,23)}$. Briefly, primers targeting all bacteria (universal primer), specific genera (Lactobacillus, Bifidobacterium, Blautia, Faecalibacterium and Fusobacterium) and specific species (Escherichia coli and Clostridium perfringens) were used. Real-time PCR conditions and primers used for Lactobacillus spp., Fusobacterium spp., Blautia spp., Faecalibacterium spp., Bifidobacterium spp. and C. perfringens have been described by Rossi et al. ${ }^{(22)}$. Real-time PCR conditions and primers for $E$. coli have been described by Malinen et al. ${ }^{(23)}$. Briefly, standard curves were generated using DNA (ranging from $2 \mathrm{ng}$ to $0.2 \mathrm{pg}$ ) from lyophilised bacterial species of the genera (Faecalibacterium prausnitzii (ATCC 27766); Lactobacillus rhamnosus GG (ATCC 53 103); Bifidobacterium bifidum (ATCC 11863); Blautia coccoides (ATCC 29236); Fusobacterium nucleatum (ATCC 25586)), clinical isolates from E. coli and C. perfringens, and canine faecal community DNA for universal bacteria. qPCR data are expressed as the log amount of DNA (fg) for each particular bacterial group/10 ng of isolated total DNA ${ }^{(24)}$.

\section{Statistical analyses}

Sequence percentages at each taxonomic level were analysed using the MIXED model procedure of SAS (version 9.2; SAS Institute, Inc.). The fixed effect of treatment was tested, and dog and period were considered as random effects. PROC UNIVARIATE was used to test for homogeneity of variance. For data with non-homogeneous variance, observed values were reported, and these data were transformed using the $\log$ function before statistical analyses. Means were separated for treatments using a Fisher-protected least significant difference with Tukey's adjustment. Chao 1 values were calculated and rarefaction curves were created to compare microbial diversity and species richness among the samples. Principal components analysis was calculated to determine the clustering effects among the diets. Results are reported as leastsquares means, with $P \leq 0.05$ being defined as significant. Means that were above or below 3 standard deviations were considered outliers and removed. Post hoc Pearson's correlations on qPCR, sequence and SCFA data were analysed by the PROC CORR procedure in SAS. Additionally, orthogonal contrasts were used to test the linear and quadratic effects of providing graded concentrations of dietary $\mathrm{PF}$, and all $\mathrm{PF}$ treatments $(1.5$ to $6 \%$ ) were compared with the $0 \% \mathrm{PF}$ control using a single df contrast.

\section{Results}

Pyrosequencing of 16S rRNA gene-barcoded amplicons resulted in a total of 1040107 sequences, with an average of 20802 reads (range $10647-56247$ ) per sample. The samples had an average read length of $542 \mathrm{bp}$. According to Chao 1 values and rarefaction curves (data not shown), microbial diversity and species richness was similar among the treatments. Principal components analysis revealed no clustering among the dietary treatments (online supplementary Fig. S1). Regardless of the dietary treatments, Firmicutes (22.63-94.21\% of all sequences) was the predominant bacterial phylum in dog faeces, followed by Fusobacteria 
Table 2. Predominant bacterial phyla and genera (expressed as a percentage of total sequences) in the faeces of dogs fed diets containing graded concentrations of potato fibre (PF)†

\begin{tabular}{|c|c|c|c|c|c|c|c|c|c|}
\hline \multirow[b]{2}{*}{ Phylum } & \multirow[b]{2}{*}{ Family } & \multirow[b]{2}{*}{ Genus } & \multicolumn{5}{|c|}{$\% \mathrm{PF}$} & \multirow[b]{2}{*}{ SEM } & \multirow[b]{2}{*}{$P$} \\
\hline & & & 0 & 1.5 & 3 & 4.5 & 6 & & \\
\hline \multirow[t]{2}{*}{ Actinobacteria } & & & $0.23^{a, b}$ & $0.27^{a, b}$ & $0.20^{a, b}$ & $0.54^{b}$ & $0 \cdot 10^{a}$ & 0.22 & 0.03 \\
\hline & Bifidobacteriaceae & Bifidobacterium & 0.44 & 0.25 & $0 \cdot 17$ & 0.52 & 0.09 & 0.23 & 0.17 \\
\hline \multirow{3}{*}{ Bacteroidetes } & & & $1 \cdot 17$ & 0.70 & 1.17 & 1.05 & 0.73 & 0.33 & 0.57 \\
\hline & Bacteroidaceae & Bacteroides & 0.39 & 0.43 & $0 \cdot 70$ & 0.47 & 0.51 & $0 \cdot 20$ & 0.72 \\
\hline & Prevotellaceae & Prevotella* & $0.78^{b}$ & $0 \cdot 27^{a}$ & $0.47^{a, b}$ & $0.59^{a, b}$ & $0 \cdot 26^{\mathrm{a}}$ & 0.42 & 0.02 \\
\hline \multirow{14}{*}{ Firmicutes } & & & $56 \cdot 66$ & 64.33 & 64.52 & $68 \cdot 20$ & $58 \cdot 17$ & 5.99 & 0.17 \\
\hline & Clostridiaceae & Clostridium§ & $26 \cdot 54^{\mathrm{b}}$ & $23 \cdot 00^{a, b}$ & $26 \cdot 96^{\mathrm{b}}$ & $26 \cdot 63^{b}$ & $14 \cdot 61^{\mathrm{a}}$ & $5 \cdot 62$ & 0.01 \\
\hline & Eubacteriaceae & Eubacterium & 0.79 & 1.27 & 0.95 & $1 \cdot 10$ & 0.57 & 0.32 & 0.46 \\
\hline & Lachnospiraceae & Blautia§ & $11 \cdot 27$ & $12 \cdot 24$ & $12 \cdot 50$ & $13 \cdot 29$ & $10 \cdot 40$ & $2 \cdot 11$ & 0.22 \\
\hline & & Lachnospira*§ & $0.02^{\mathrm{a}}$ & $0.92^{a, b}$ & $1.52^{\mathrm{b}}$ & $1.57^{\mathrm{b}}$ & $1.51^{\mathrm{b}}$ & 0.42 & 0.01 \\
\hline & & Roseburia & 0.83 & 0.70 & 0.29 & 0.76 & 0.33 & 0.27 & 0.37 \\
\hline & Lactobacillaceae & Lactobacillus & 1.27 & $5 \cdot 26$ & 1.99 & 2.94 & 4.46 & $2 \cdot 43$ & 0.71 \\
\hline & Peptococcaceae & Desulfotomaculum & 1.26 & 1.26 & 0.84 & 0.70 & 0.88 & 0.40 & 0.48 \\
\hline & Ruminococcaceae & Faecalibacterium $^{\star} \ddagger$ & $4 \cdot 64^{a}$ & $9 \cdot 38^{a}$ & $9 \cdot 57^{\mathrm{a}}$ & $10 \cdot 23^{a, b}$ & $15 \cdot 69^{b}$ & 2.07 & 0.01 \\
\hline & & Oscillospira & 0.72 & 0.76 & 0.71 & 0.72 & 0.46 & $0 \cdot 19$ & 0.60 \\
\hline & & Ruminococcus & 5.49 & $5 \cdot 26$ & 4.63 & 4.93 & 4.72 & 1.05 & 0.86 \\
\hline & Turcibacteraceae & Turicibacter & 0.89 & 0.67 & 0.96 & 1.02 & 0.66 & 0.27 & 0.49 \\
\hline & Veillonellaceae & Megamonas & 0.62 & 0.71 & $0 \cdot 80$ & 1.03 & 0.96 & 0.38 & 0.93 \\
\hline & & Phascolarctobacterium & $3 \cdot 30$ & 3.57 & 3.65 & 3.45 & 3.49 & 0.85 & 0.99 \\
\hline \multirow[t]{2}{*}{ Fusobacteria§ } & & & $27 \cdot 44$ & $25 \cdot 84$ & 23.01 & $20 \cdot 29$ & $32 \cdot 28$ & $4 \cdot 80$ & 0.17 \\
\hline & Fusobacteriaceae & Fusobacterium§ & $27 \cdot 44$ & $25 \cdot 84$ & 23.01 & $20 \cdot 29$ & $32 \cdot 28$ & $4 \cdot 80$ & 0.17 \\
\hline \multirow{3}{*}{ Proteobacteria } & & & 1.53 & 1.77 & 1.80 & $2 \cdot 72$ & $2 \cdot 78$ & 0.83 & 0.62 \\
\hline & Succinivibrionaceae & Succinivibrio* $\ddagger$ & 0.08 & 0.23 & $0 \cdot 19$ & $0 \cdot 31$ & 0.29 & $0 \cdot 11$ & 0.18 \\
\hline & Alicaligenaceae & Sutterella & 1.39 & 1.53 & 1.52 & $2 \cdot 00$ & 2.43 & $0 \cdot 77$ & 0.82 \\
\hline \multirow[t]{6}{*}{ Tenericutesł } & & & $12 \cdot 74$ & $7 \cdot 09$ & $9 \cdot 29$ & $7 \cdot 20$ & $5 \cdot 20$ & $2 \cdot 52$ & 0.18 \\
\hline & Erysipelotrichaceae & Allobaculum & $10 \cdot 52$ & $4 \cdot 31$ & $6 \cdot 38$ & $5 \cdot 28$ & 3.57 & $2 \cdot 55$ & 0.67 \\
\hline & & Bulleidia & 0.21 & 0.18 & 0.22 & 0.15 & 0.16 & 0.06 & 0.57 \\
\hline & & Catenibacterium & 0.14 & 0.12 & 0.07 & 0.07 & 0.04 & 0.06 & 0.68 \\
\hline & & Coprobacillus§ & 0.38 & 0.51 & 0.51 & 0.45 & 0.25 & $0 \cdot 13$ & 0.26 \\
\hline & & Holdemania & 0.07 & $0 \cdot 10$ & 0.08 & 0.08 & 0.04 & 0.04 & 0.59 \\
\hline
\end{tabular}

${ }^{a, b}$ Mean values within a row with unlike superscript letters were significantly different $(P<0.05)$.

* Mean value was significantly different from that of the $0 \%$ PF diet $(P<0.05)$.

t Genera included had a least-squares mean value of 0.01 or higher.

† Linear effect $(P<0.05)$.

$\S$ Quadratic effect $(P<0.05)$.

(0.53-58.18\% of all sequences) and Tenericutes (1.78-40.96\% of all sequences) (Table 2). Actinobacteria ( $0-3 \cdot 47 \%$ of all sequences), Bacteroidetes (0-9.06\% of all sequences) and Proteobacteria $(0-12.53 \%$ of all sequences) also were present. Inclusion of graded concentrations of dietary PF in canine diets altered the proportions of bacterial phyla in the canine faecal microbiome. With increasing PF concentrations, the proportion of Firmicutes exhibited a positive quadratic response $(P<0.05)$, with the highest concentration observed in dogs fed the $4.5 \%$ PF diet. Fusobacteria exhibited a negative quadratic response $(P<0.05)$, with the lowest concentration observed in dogs fed the $4.5 \% \mathrm{PF}$ diet. The proportion of Tenericutes decreased linearly $(P<0.05)$ with increasing dietary PF concentrations.

Fusobacterium (0.53-58.18\% of all sequences), Clostridium (3.21-61.35\% of all sequences), Blautia (1.77-26.38\% of all sequences) and Faecalibacterium (0.20-27.99\% of all sequences) were the predominant genera in dog faeces (Table 2). The proportion of Fusobacterium exhibited a negative quadratic response $(P<0 \cdot 05)$, with the lowest concentrations observed in dogs fed the $4.5 \% \mathrm{PF}$ diet. Within the phylum Firmicutes, Clostridium was the predominant genera for dogs fed 0 to $4.5 \%$ PF. For dogs fed the $6.0 \% \mathrm{PF}$ diet, the proportion of Clostridium was lower $(P<0.05)$ than that for dogs fed the 0,3 or $4.5 \%$ PF diet, while the proportion of Faecalibacterium was greater $(P<0.05)$ than that for dogs fed the $0,1.5$ or $3 \% \mathrm{PF}$ diet. The proportion of Clostridium exhibited a quadratic response $(P<0.05)$ with increasing dietary $\mathrm{PF}$ concentrations, with dogs fed $6 \% \mathrm{PF}$ having lower proportions $(P<0.05)$ than those fed the $0 \%$ PF diet. The proportions of Faecalibacterium and Lachnospira were greater $(P<0.05)$ for dogs fed all diets containing PF compared with those fed the $0 \% \mathrm{PF}$ diet. The proportion of Faecalibacterium increased linearly $(P<0.05)$, while that of Lachnospira increased quadratically $(P<0 \cdot 05)$, with increasing dietary $P F$ concentrations. The proportions of Blautia increased quadratically $(P<0.05)$, with peak proportions observed when dogs were fed the $4.5 \%$ PF diet. For the faecal proportion of Coprobacillus, there was also a quadratic increase $(P<0.05)$, with peak proportions being observed in dogs fed the 1.5 and $3 \% \mathrm{PF}$ diets. The proportions of Prevotella (member of Bacteroidetes) and Succinivibrio (member of Proteobacteria) were lower $(P<0.05)$ for dogs fed all PF-containing diets compared with those fed the $0 \%$ $\mathrm{PF}$ diet, and also exhibited a linear decrease $(P<0.05)$ with increasing dietary PF concentrations. Bacterial species 
(a)

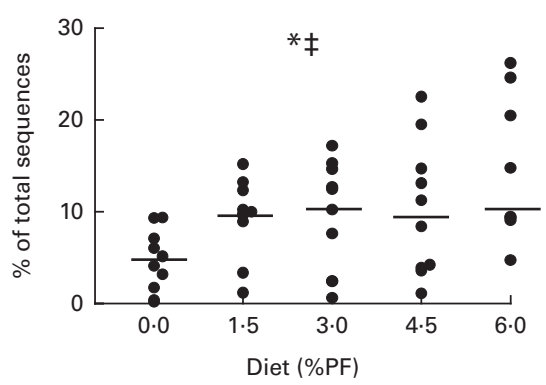

(d)

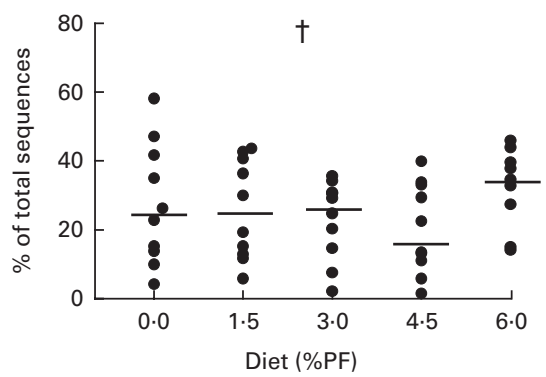

(b)

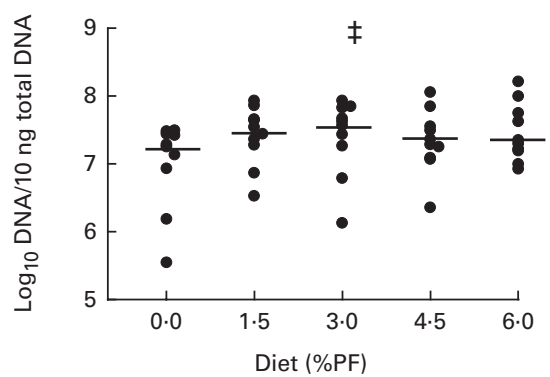

(e)

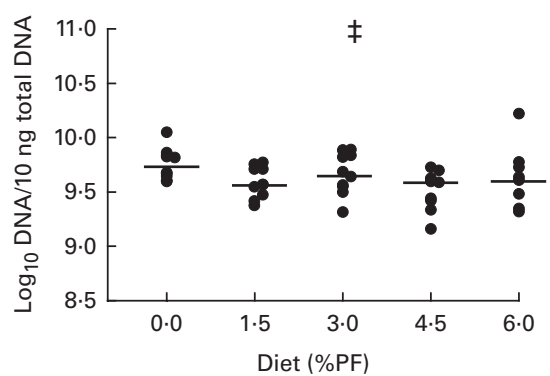

(h)

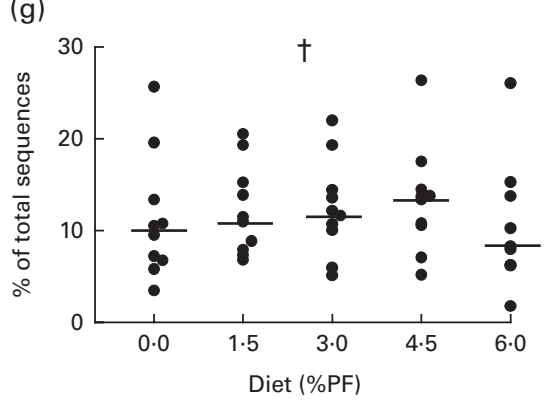

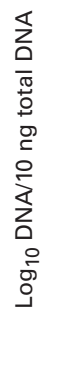

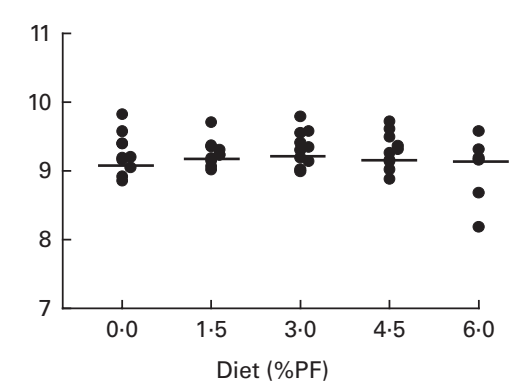

(c)

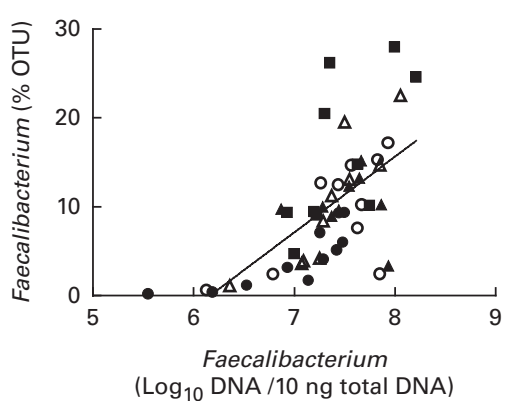

(f)

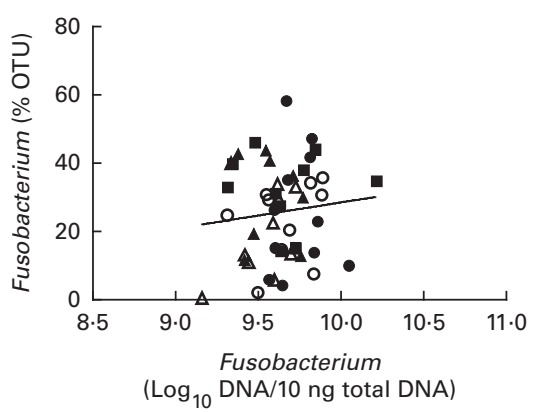

(i)

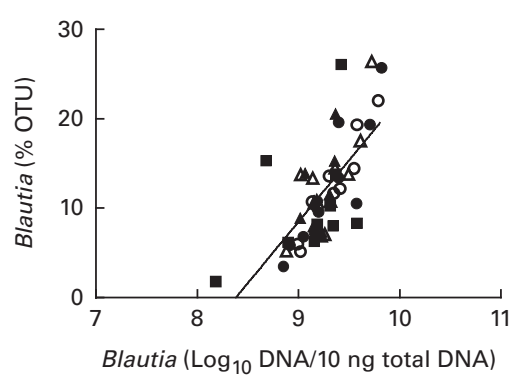

Fig. 1. Faecalibacterium spp. scatter plots for sequencing data (a), quantitative PCR (qPCR) data (b), and Pearson's correlation analysis for sequence $v$. qPCR data $(R 0.64 ; P \leq 0.01)$ (c). Fusobacterium spp. scatter plots for sequencing data (d), qPCR data (e), and Pearson's correlation analysis for sequence $v$. qPCR data $(R 0.10 ; P=0.44)(\mathrm{f})$. Blautia spp. scatter plots for sequencing data $(\mathrm{g})$, qPCR data $(\mathrm{h})$, and Pearson's correlation analysis for sequence $v$. qPCR data $(R$ 0.69; $P \leq 0.01)$ (i). * Least-squares means show a linear effect $(P<0.05)$. † Least-squares means show a quadratic effect $(P<0.05)$. $\neq$ Least-squares means show a difference between $0 \%$ potato fibre (PF) v. all other PF diets $(P<0.05)$. Pearson's correlation plots: $\bullet, 0 \%$ PF diet; $\mathbf{\Lambda}, 1.5 \%$ PF diet; $\bigcirc, 3 \%$ PF diet; $\Delta, 4.5 \%$ PF diet; $\mathbf{\square}, 6$ PF diet. OTU, operational taxonomic units.

also were quantified and are presented in online supplementary Table S1.

qPCR was conducted on select genera and species. Fig. 1 shows the scatter plots of sequence and qPCR data with median values, and Pearson's correlation scatter plots of qPCR $v .454$ pyrosequencing for Faecalibacterium spp. (Fig. 1(a)-(c)), Fusobacterium spp. (Fig. 1(d)-(f)) and Blautia spp. (Fig. 1(g)-(i)), respectively. Faecal Faecalibacterium, Fusobacterium and Blautia spp. showed no significant diet effects with increasing concentrations of $\mathrm{PF}$ in the diet $(P=0.10,0.07$ and 0.32 , respectively; means are not shown). A single df contrast of the qPCR data calculating the difference between $0 \% \mathrm{PF} v$. all the other diets containing PF revealed that the faecal proportion of Faecalibacterium spp. was higher $(P<0.05)$ in dogs fed the PF-containing diets than in those fed the $0 \% \mathrm{PF}$ diet. The proportion of faecal Fusobacterium spp. was lower $(P<0.05)$ for dogs fed the PF-containing diets than for those fed the $0 \% \mathrm{PF}$ diet. There was a positive correlation between the sequence and qPCR data for Faecalibacterium spp. ( $R$ 0.64; $P<0.01)$ and Blautia spp. ( $R$ 0.69; $P<0 \cdot 01)$. There was no correlation between sequence and qPCR data for Fusobacterium spp. $(R 0 \cdot 10 ; P<0 \cdot 44)$.

Scatter plots of qPCR data with median values for Bifidobacterium and Lactobacillus spp., and Pearson's correlation plots for Bifidobacterium spp. and butyrate production and for Lactobacillus spp. and Bifidobacterium spp. are shown in Fig. 2(a)-(d). There were no differences in mean values (data not shown) for Bifidobacterium spp. and Lactobacillus spp. among the dietary treatments; however, there were significant correlations between Bifidobacterium spp. and butyrate production $(R 0.49 ; P<0.01)$ and Lactobacillus and Bifidobacterium spp. $(R \quad 0.82 ; P<0.01)$. Scatter plots for $E$. coli and $C$. perfringens $16 \mathrm{~S}$ are presented in online supplementary Fig. S1. 
(a)

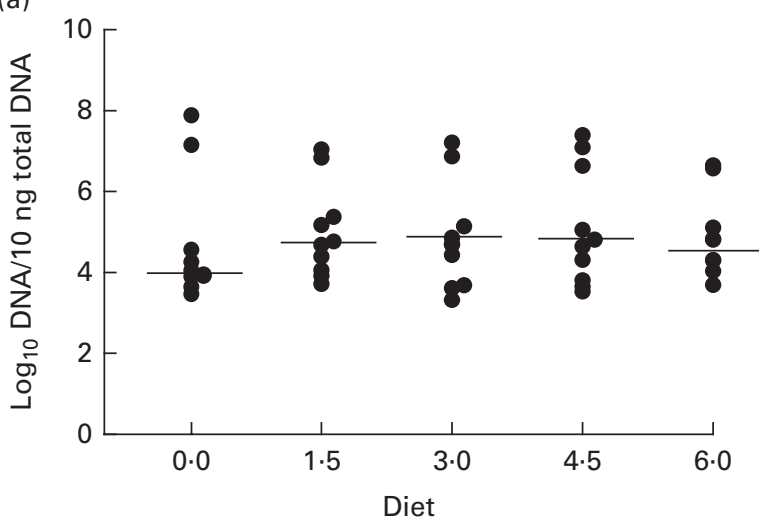

(c)

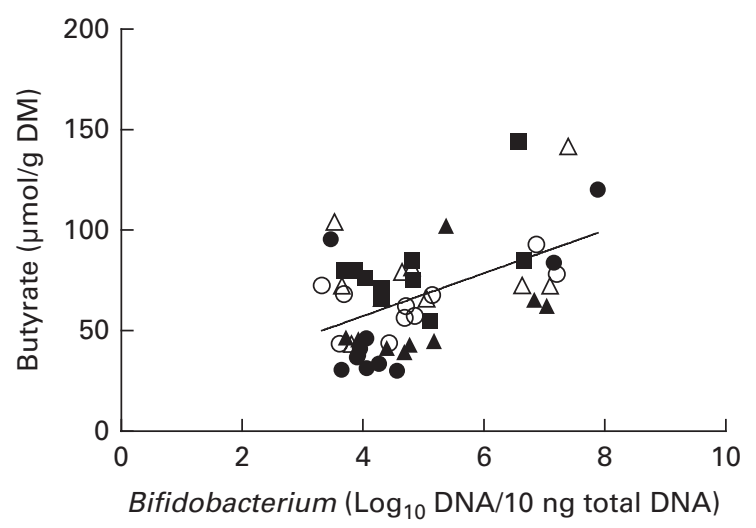

(b)

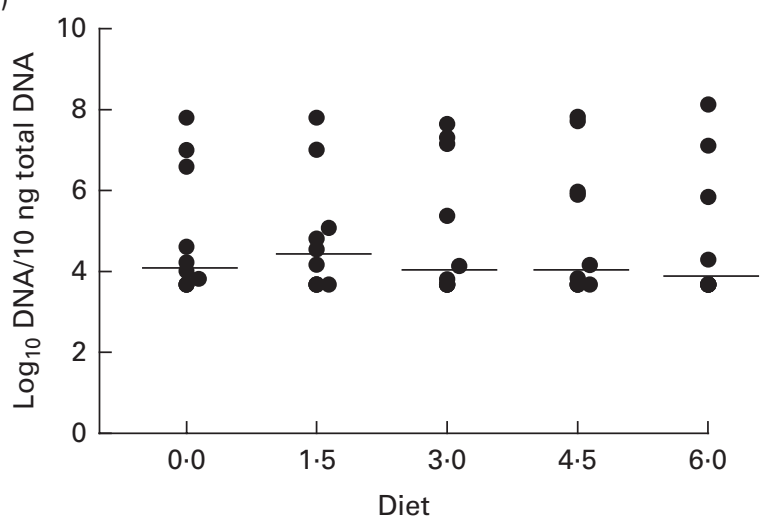

(d)

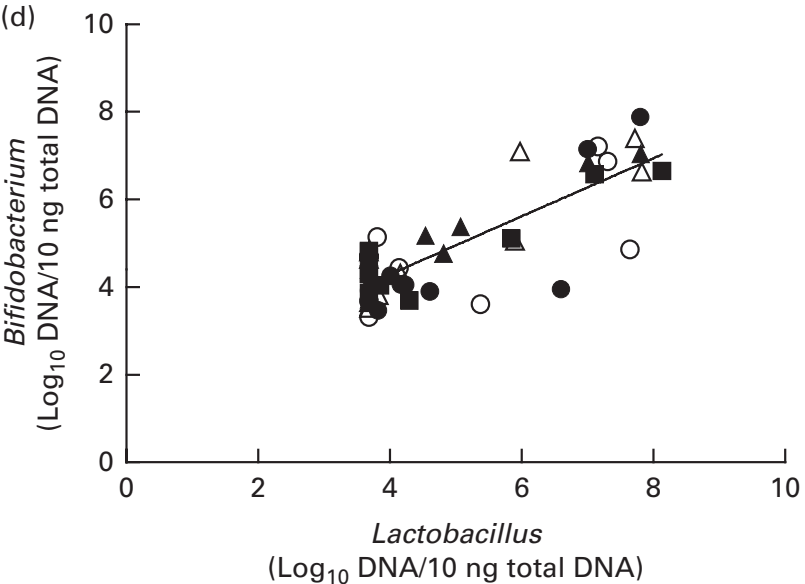

Fig. 2. Scatter plots of quantitative PCR (qPCR) data for Bifidobacterium (a) and Lactobacillus spp. (b). Data are presented as replicate values, with a line representing the median of each treatment group. Pearson's correlation plots for Bifidobacterium spp. qPCR data and butyrate $(R 0.49 ; P<0.01)(\mathrm{c})$ and Lactobacillus spp. qPCR data and Bifidobacterium spp. $(R$ 0.82; $P<0.01)$ (d). Pearson's correlation plots: $\bullet, 0 \%$ potato fibre (PF) diet; $\mathbf{\Lambda}, 1.5 \%$ PF diet; $\bigcirc, 3 \%$ PF diet; $\Delta, 4.5 \%$ PF diet; $\mathbf{\square}, 6 \%$ PF diet.

\section{Discussion}

Part 1 of the present study investigated the viability of PF as a potential dietary fibre source in dog foods by evaluating (1) the fermentability of PF in vitro and (2) nutrient digestibility, faecal consistency and fermentability of graded concentrations of dietary $\mathrm{PF}$ in vivo ${ }^{(10)}$. The results from part 1 indicated that $\mathrm{PF}$ was fermentable through $9 \mathrm{~h}$ of in vitro fermentation, and showed linear increases in the levels of faecal acetate, propionate and butyrate with increasing dietary PF concentrations ${ }^{(10)}$. It was concluded that PF was moderately fermentable and showed no detrimental effects on total tract nutrient digestibilities or faecal consistency ${ }^{(10)}$. The increased concentrations of faecal SCFA and, in particular, butyrate, as well as decreases in faecal $\mathrm{pH}$, were observed as being a benefit to host health. It is well known that SCFA have numerous benefits to the host, including improved gut health ${ }^{(25)}$, satiety promotion $^{(26)}$ and attenuated inflammation ${ }^{(27)}$. Furthermore, a decrease in faecal $\mathrm{pH}$ with increasing concentrations of dietary PF suggests carbohydrate fermentation throughout the entire length of the colon. After observing these beneficial changes in part 1 , the objective of part 2 of the present study was to identify microbial taxa altered by the graded concentrations of dietary PF.
There is limited research investigating the use of PF as a dietary fibre source. The fibre fraction of PF consists of $55 \%$ total dietary fibre, with $32 \%$ insoluble fibre and $23 \%$ soluble fibre. Specifically, the insoluble fibre fraction is predominantly cellulose (16\%) and hemicellulose (9\%). Pectin is the main component of the soluble fibre fraction of PF. A study in rats has reported that the soluble fibre fraction of PF resulted in higher caecal SCFA concentrations compared with rats fed a non-fermentable cellulose control ${ }^{(9)}$. Research in human subjects has indicated that consumption of enzymatically solubilised PF elicited increased fermentation by increasing breath $\mathrm{H}_{2}$ compared with consumption of oat bran ${ }^{(8)}$. Furthermore, in vitro data using human faecal inoculum indicated that solubilised PF that was minimally treated enzymatically was more bifidogenic than fructo-oligosaccharides ${ }^{(7)}$. The chemical composition, in vitro fermentation and in vivo responses suggest that $\mathrm{PF}$ has prebiotic potential; however, the effects of graded concentrations of dietary PF on the hindgut microbiome have yet to be studied.

Previous studies have investigated the modulatory effects of fibre on the hindgut microbiome in both dogs and human subjects $^{(4,5)}$. We concluded from our previous work that PF is moderately fermentable in vitro and in vivo ${ }^{(10)}$. Therefore, our diet formulation sought to keep total dietary fibre con- 
centrations constant among all diets, while only changing the amount of PF at the expense of an inert fibre, cellulose. With this unique diet formulation, the present study is the first to use high-throughput $16 \mathrm{~S}$ rRNA gene-based pyrosequencing to determine how increasing concentrations of a moderately fermentable fibre modulates the hindgut microbiota of dogs.

Previous data indicated that the faecal microbiome of a healthy dog consists of approximately 14 to $52.5 \%$ Firmicutes and 7 to $50 \%$ Fusobacteria ${ }^{(5,20,28,29)}$. The predominant bacterial phyla present in the faeces of dogs fed all diets were Firmicutes and Fusobacteria. The order of predominant faecal bacterial phyla found in the present study was different from that reported in other studies investigating the dog faecal microbiome. These differences could be explained by the variable regions of the $16 \mathrm{~S}$ rRNA being amplified. For example, Middelbos et al. ${ }^{(5)}$ found healthy dogs to have predominantly Fusobacteria, followed by Firmicutes and Bacteroides, when amplifying the V3 region of 16S rRNA. Similarly, studies investigating the V1-V3 regions of $16 \mathrm{~S}$ rRNA found very high proportions of Firmicutes ( $96 \%$ OTU) and very low proportions of other bacterial phyla. The present study investigated the V4-V6 regions of $16 \mathrm{~S}$ bacterial rRNA, which resulted in different proportions of predominant bacterial phyla; however, the shifts in the microbiome are consistent with fibre supplementation ${ }^{(5)}$. The supplementation of beet pulp has been found to increase the ratio of Firmicutes: Fusobacteria $^{(5)}$. A similar pattern was found in the present study where increasing dietary PF concentrations elicited a quadratic increase in the proportion of faecal Firmicutes and a quadratic decrease in the proportion of faecal Fusobacteria.

Fermentability of fibrous substrates is dependent on the chemical composition, physical form and amount of the substrate available ${ }^{(30)}$. The heat, pressure and moisture applied in extrusion usually increases the availability of the substrate for microbes to ferment and produce SCFA. Typically, the molar concentrations of SCFA in the hindgut are greatest in acetate, then propionate, and butyrate often is present in the lowest concentration. Butyrate is a major energy source for colonocytes, and promotes cell apoptosis, stimulation of cell proliferation, and prevention of colon cancer ${ }^{(31)}$. Part 1 of the present study found increases in the concentrations of all the three SCFA with increasing dietary PF concentrations. Changes within the phylum Firmicutes with increasing PF concentrations are consistent with the present results on faecal SCFA concentrations, and provide some insight into the physiological relevance of the present study.

Diversity within the phylum Firmicutes is indicative of a reduced risk of certain hindgut diseases, as well as concentrations of a diet containing fermentable carbohydrates ${ }^{(4,5,32)}$. Increases in butyrate and other SCFA concentrations also may be revealing of shifts in the hindgut microbiome ${ }^{(25)}$. The present results did not show increased diversity within the phylum Firmicutes; however, we showed an increase in the abundance of key fermenters. We showed an increase in the proportion of Faecalibacterium spp. with increasing dietary PF concentrations, which is consistent with previous studies examining fermentable fibre in dog and human $\operatorname{diets}^{(4,5)}$. Dogs ingesting beet pulp, a moderately fermentable fibre with a similar chemical composition to PF, were found to have more amounts of $F$. prausnitzii represented in faecal samples compared with dogs provided no fibre ${ }^{(5)}$. This bacterium has received increased attention due to the human literature suggesting its protective effects on inflammatory bowel disease $^{(33,34)}$. In dogs, it has been generally accepted as a beneficial bacterium due to its depletion in inflammatory bowel disease $^{(24)}$ and its ability to produce butyrate ${ }^{(35)}$; however, its physiological relevance has not been fully elucidated. In the present study, the GPCR analysis of this bacterium confirmed its presence, and the analysis was highly correlated with our sequencing data. Furthermore, both the sequencing and qPCR data showed similar patterns of increased proportions of Faecalibacterium with increasing dietary PF concentrations.

Faecal Blautia and Fusobacterium spp. were two genera that were abundant in our sequencing data and were confirmed by qPCR. There was a significant positive correlation between qPCR and sequencing data when analysing faecal Blautia spp., but no significant correlation was observed with Fusobacterium spp. This result was not entirely surprising, since our previous publications using these primers tended to have a bias towards Fusobacterium spp. ${ }^{(5,20)}$. The qPCR data did not show significant diet effects; however, the pattern observed with increasing $\mathrm{PF}$ concentrations seemed to be similar to the sequence data. In dogs, low faecal concentrations of Blautia spp. have been associated with acute-haemorrhagic diarrhoea, are acetogens and are a part of the butyrate-producing superfamily Lachnospiraceae. Fusobacterium spp. are largely proteolytic bacteria and have previously been reported to have associations with liver cirrhosis $^{(36)}$ and oral cavity infections in humans ${ }^{(37)}$; however, the presence of this bacterial phylum is common in healthy dogs ${ }^{(5,26,27)}$. An increase in the proportions of faecal Blautia spp. and a decrease in the proportions of faecal Fusobacterium spp. are often observed with dietary fibre intake ${ }^{(5)}$.

The physiological relevance of these results was further investigated by conducting post hoc Pearson's correlation analysis with faecal SCFA data reported previously ${ }^{(14)}$ and qPCR data. Prebiotics are often evaluated by their ability to produce butyrate (a butyrogenic effect) or to stimulate the growth of bifidobacteria (a bifidogenic effect); however, the definition of a prebiotic is not limited to this genus ${ }^{(38,39)}$. We found that Bifidobacterium spp. were positively correlated with the concentrations of butyrate and lactobacilli. The main carbohydrate fermentation products of Bifidobacteria are acetate and lactate when the substrate is available ${ }^{(29)}$, while lactobacilli produce lactic acid. Bifidobacterial growth has been found to accompany butyrate production with inulin-type fructans ${ }^{(38)}$. This has previously been explained by bacterial cross-feeding where primary fermenters of carbohydrate will produce secondary metabolites (acetate and lactate) or hydrolysed substrate that can be used by other bacteria $^{(38)}$. Pectic oligosaccharides have been found to increase bifidobacteria in vitro; however, the effect is dependent on the degree of methylation, and it was concluded that oligo-fructose was more bifidogenic ${ }^{(40)}$. An in vitro fermentation study using human faecal inoculum found that solubilised PF that had minimal enzymatic treatment was 
more bifidogenic than fructo-oligosaccharides ${ }^{(7)}$. The positive correlations between Bifidobacterium spp. with butyrate production and Lactobacillus growth further suggest the prebiotic potential of PF; however, the bifidogenic properties of PF need to be investigated further.

In conclusion, increasing concentrations of dietary PF were found to modulate the faecal microbiome at the phylum and genus taxonomic levels. This was confirmed by qPCR where faecal Faecalibacterium and Blautia spp. showed significant positive correlations with 454 pyrosequencing data. Furthermore, part 1 of the present study found increases in the levels of faecal acetate, propionate and butyrate with increasing dietary PF concentrations, which appears to agree with the changes in the faecal microbiome. Post hoc Pearson's correlation analysis also showed significant correlations between Bifidobacterium spp. with butyrate production and Lactobacillus spp. concentrations. One potential pitfall of the present study was the use of BLAST to infer taxonomy rather than using a Ribosomal Database Project (RDP) classifier. Although some may prefer the use of RDP, small differences have been previously observed to exist between RDP and BLAST at higher taxonomic levels ${ }^{(41)}$. Inferences in the present study were only made on the sequences of the bacterial group confirmed by qPCR data and at the phylum and genus taxonomic levels where such differences should have been rather small. Overall, the present study suggests that $\mathrm{PF}$ is beneficial to gut health and should be investigated as a novel functional ingredient in dog diets. Future research investigating the effects of other novel fibre sources on the microbiome could help explain how different clusters of bacterial families and genera respond to fermentable substrates.

\section{Supplementary material}

To view supplementary material for this article, please visit http://dx.doi.org/10.1017/S0007114514003274

\section{Acknowledgements}

The present study was supported by Roquette Frères, Biology and Nutrition Department.

The authors' contributions were as follows: M. R. P., K. R. K., R. N. D., G. C. F., L. G.-D., G. L. L., D. W., S. E. D. and K. S. S. designed the research; M. R. P. and K. R. K. conducted the research; M. R. P., K. R. K. and S. E. D. analysed the data; M. R. P. and K. S. S. wrote the paper; K. S. S. had primary responsibility for the final content of the manuscript. All authors read and approved the final manuscript.

L. G.-D., G. L. L. and D. W. are co-authors who are employees of Roquette Frères, Biology and Nutrition Department. The rest of the authors have no conflicts of interest to declare.

\section{References}

1. Khachatryan ZA, Ktsoyan ZA, Manukyan GP, et al. (2008) Predominant role of host genetics in controlling the composition of gut microbiota. PLOS ONE 3, e3064.
2. Mariat D, Firmesse O, Levenez F, et al. (2009) The Firmicutes/Bacteroidetes ratio of the human microbiota changes with age. BMC Microbiol 9, 123-128.

3. Yatsunenko T, Rey FE, Manary MJ, et al. (2012) Human gut microbiome viewed across age and geography. Nature 486, 222-227.

4. Hooda S, Boler BM, Serao MC, et al. (2012) 454 Pyrosequencing reveals a shift in fecal microbiota of healthy adult men consuming polydextrose or soluble corn fiber. J Nutr $\mathbf{1 4 2}$, $1259-1265$

5. Middelbos IS, Vester Boler BM, Qu A, et al. (2010) Phylogenetic characterization of fecal microbial communities of dogs fed diets with or without supplemental dietary fiber using 454 pyrosequencing. PLOS ONE 5, e9768.

6. Mayer F (1998) Potato pulp: properties, physical modification and applications. Polym Degrad Stab 59, 231-235.

7. Thomassen LV, Vigsnaes LK, Licht TR, et al. (2011) Maximal release of highly bifidogenic soluble dietary fibers from industrial potato pulp by minimal enzymatic treatment. Appl Microbiol Biotechnol 90, 873-884.

8. Olesen M, Gudmand-Hoyer E, Norsker M, et al. (1998) Fermentability of an enzymatically modified solubilised potato polysaccharide (SPP). Eur J Clin Nutr 52, 110-114.

9. Laerke HN, Meyer AS, Kaack KV, et al. (2007) Soluble fiber extracted from potato pulp is highly fermentable but has no effect on risk markers of diabetes and cardiovascular disease in Goto-Kakizaki rats. Nutr Res 27, 152-160.

10. Panasevich MR, Rossoni Serao MC, de Godoy MRC, et al. (2013) Potato fiber as a dietary fiber source in dog foods. J Anim Sci 91, 5344-5352.

11. NRC (2006) Nutrient Requirements of Dogs and Cats. Washington, DC: National Academy Press.

12. McInnes P \& Cutting M (2010) Manual of procedures for human microbiome project: core microbiome sampling, protocol A, HMP protocol no. 07-001, version 11. www.hmpdacc.org/doc/sops_2/manual_of_procedures_v11.pdf

13. Cephas KD, Kim J, Mathai RA, et al. (2011) Comparative analysis of salivary bacterial microbiome diversity in edentulous infants and their mothers or primary care givers using pyrosequencing. PLOS ONE 6, e23503.

14. Dowd SE, Callaway TR, Wolcott RD, et al. (2008) Evaluation of the bacterial diversity in the feces of cattle using $16 \mathrm{~S}$ rDNA bacterial tag-encoded FLX amplicon pyrosequencing (bTEFAP). BMC Microbiol 8, 125-141.

15. Dowd SF, Sun Y, Wolcott RD, et al. (2008) Bacterial tag-encoded FLX amplicon pyrosequencing (bTEFAP) for microbiome studies: bacterial diversity in the ileum of newly weaned Salmonella-infected pigs. Foodborne Pathog Dis 5, 459-472.

16. Edgar RC (2010) Search and clustering orders of magnitude faster than BLAST. Bioinformatics 26, 2460-2461.

17. Capone KA, Dowd SE, Stamatas GN, et al. (2011) Diversity of the human skin microbiome early in life. J Invest Dermatol 131, 2026-2032.

18. Dowd SE, Hanson JD, Rees E, et al. (2011) Survey of fungi and yeast in polymicrobial infections in chronic wounds. $J$ Wound Care 20, 40-47.

19. Eren AM, Zozaya M, Taylor CM, et al. (2011) Exploring the diversity of Gardnerella vaginalis in the genitourinary tract microbiota of monogamous couples through subtle nucleotide variation. PLOS ONE 6, e26732.

20. Swanson KS, Dowd SE, Suchodolski JS, et al. (2011) Phylogenetic and gene-centric metagenomics of the canine intestinal microbiome reveals similarities with humans and mice. ISME J 5, 639-649. 
21. DeSantis TZ, Hugenholtz P, Larsen N, et al. (2006) Greengenes, a chimera-checked 16S rRNA gene database and workbench compatible with ARB. Appl Environ Microbiol 72, 5069-5072.

22. Rossi G, Pengo G, Caldin M, et al. (2014) Comparison of microbiological, histological, and immunomodulatory parameters in response to treatment with either combination therapy with prednisone and metronidazole or probiotic VSL\#3 strains in dogs with idiopathic inflammatory bowel disease. PLOS ONE 9, e94699.

23. Malinen E, Rinttila T, Kajander K, et al. (2005) Analysis of the fecal microbiota of irritable bowel syndrome patients and healthy controls with real-time PCR. Am J Gastroenterol 100, 373-382.

24. Suchodolski JS, Markel ME, Garcia-Mazcorro JF, et al. (2012) The fecal microbiome in dogs with acute diarrhea and idiopathic inflammatory bowel disease. PLOS ONE 7, e51907.

25. Wong JM, de Souza R, Kendall CW, et al. (2006) Colonic health: fermentation and short chain fatty acids. $J$ Clin Gastroenterol 40, 235-243.

26. Tolhurst G, Heffron H, Lam YS, et al. (2012) Short-chain fatty acids stimulate glucagon-like peptide-1 secretion via the G-protein-coupled receptor FFAR2. Diabetes $\mathbf{6 4}$, 364-371.

27. Vinolo MA, Rodrigues HG, Nachbar RT, et al. (2011) Regulation of inflammation by short chain fatty acids. Nutrients 3, 858-876.

28. Handl S, German AJ, Holden SE, et al. (2013) Fecal microbiota in lean and obese dogs. FEMS Microbiol Ecol 84, $332-343$.

29. Suchodolski JS, Camacho J \& Steiner JM (2008) Analysis of bacterial diversity in the canine duodenum, jejunum, ileum, and colon by comparative 16S rRNA gene analysis. FEMS Microb Ecol 66, 567-578.

30. Macfarlane S \& Macfarlane GT (2003) Regulation of shortchain fatty acid production. Proc Nutr Soc 62, 67-72.
31. Hamer HM, Jonkers D, Venema K, et al. (2008) Review Article: the role of butyrate on colonic function. Aliment Pharmacol Ther 27, 104-119.

32. Manichanh C, Rigottier-Gois L, Bonnaud E, et al. (2006) Reduced diversity of faecal microbiota in Crohn's disease revealed by a metagenomic approach. Gut 55, 205-211.

33. Sokol H, Pigneur B, Watterlot L, et al. (2008) Faecalibacterium prausnitzii is an anti-inflammatory commensal bacterium identified by gut microbiota analysis of Crohn disease patients. Proc Natl Acad Sci $U$ S A 105, 16731-16736.

34. Ramirez-Farias C, Slezak K, Fuller Z, et al. (2009) Effect of inulin on the human gut microbiota: stimulation of Bifidobacterium adolescentis and Faecalibacterium prausnitzii. Br J Nutr 101, 541-550.

35. Louis P, Young P, Holtrop G, et al. (2010) Diversity of human colonic butyrate-producing bacteria revealed by analysis of the butyryl-CoA:acetate CoA-transferase gene. Environ Microbiol 12, 304-314.

36. Swidsinski A, Dorffel Y, Loening-Baucke V, et al. (2011) Acute appendicitis is characterised by local invasion with Fusobacterium nucleatum/necrophorum. Gut $\mathbf{6 0}$, 34-40.

37. Bennett KW \& Eley A (1993) Fusobacteria: new taxonomy and related diseases. J Med Microbiol 39, 246-254.

38. De Vuyst L \& Leroy F (2011) Cross-feeding between bifidobacteria and butyrate-producing colon bacteria explains bifidobacterial competitiveness, butyrate production, and gas production. Int J Food Microbiol 149, 73-80.

39. Tremaroli V \& Backhed F (2012) Functional interactions between the gut microbiota and host metabolism. Nature 489, 242-249.

40. Olano-Martin E, Gibson GR \& Rastell RA (2002) Comparison of the in vitro bifidogenic properties of pectins and pecticoligosaccharides. J Appl Microbiol 93, 505-511.

41. Jumpstart Consortium Human Microbiome Project Data Generation Working Group (2012) Evaluation of 16S rDNAbased community profiling for human microbiome research. PLOS ONE 7, e39315. 